\title{
Medientransparenz als Bildungsaufgabe
}

\author{
von Rainald Merkert
}

In seiner abendfüllenden Jubiläumssendung am 16. Dezember 1985 brachte das Dritte Fernsehprogramm des WDR einen Rückblick auf zwanzig Jahre Programmentwicklung. Werner Höfer selbst, einstiger Direktor und geistiger Vater des »wdr Westdeutsches Fernsehen ", spann gemeinsam mit Hans Janke, Leiter des Adolf-Grimme-Instituts des Deutschen Volkshochschulverbandes, den Faden der Erinnerung. Auch die einstige Sendereihe»Glashaus «zogen sie in ihre Retrospektive ein. Daß Janke sich dabei nicht mit dem bloBen Blick zurück begnügte, sondern für deren Erneuerung plädierte, mag man noch als eine Pflichtübung registrieren; daß Höfer ihm nachdrücklich beipflichtete, daß er also auch heute noch an dieser Programmidee festhält, verdient jedoch festgehalten zu werden.

Nun soll hier nicht zum wiederholten Male ein Nachruf auf jene Sendereihe angestimmt werden, die nach einer Phase des Experimentierens im September 1971 etabliert wurde, von der es damals im ARD-Jahrbuch hieß, sie stelle den ersten regelmäßigen Versuch dar, »ein Fernsehprogramm durchsichtig zu machen«; die sich bei Medienexperten und Kritikern großen Wohlwollens erfreute, beim Publikum hingegen kaum ankam, und die nach Höfers Ausscheiden aus dem WDR zunächst noch eine zeitlang dahinexistierte, zum Jahresende 1983 aber endgültig eingestellt wurde. Sie soll hier vielmehr als Exempel dienen für eine verpaßte Chance.

\section{Kritik statt Transparenz - ein Rückblick}

Warum wurde diese Chance nicht genutzt, oder richtiger, warum wurde sie verspielt? Offenbar war nicht der Mangel an Engagement und gutem Willen die Ursache, es lag vielmehr an der Art des Vorgehens, an den eingeschlagenen Wegen, vor allem aber an der Konkretisierung der Zielsetzungen. Diese Ursachen waren zwar damals schon erkennbar, aus der zeitlichen Distanz aber werden sie noch deutlicher; überdies müßte man inzwischen in der Lage sein, ohne Voreingenommenheiten und Emotionen der Frage nachzugehen, warum diese Programmidee gescheitert ist.

Das Glashaus litt von Anfang an unter einem entscheidenden Konstruktionsfehler, den jedoch weder seine Planer und Realisatoren, noch die es mit ungewöhnlicher Sympathie begleitenden Experten wahrhaben wollten: es war gar kein »Glashaus«. Wer diesen Titel mit Transparenz, mit »Durchsichtigmachen des Fernsehprogramms « in Verbindung brachte, wer ihn also beim Wort nahm, der wartete stets vergebens auf die Einlösung des Titelversprechens. Und die verantwortlichen Redakteure machten auch gar keinen Hehl daraus, daß es ihnen um Kritik des eigenen Mediums ging, aber nicht um dessen Transparenz, allen Verlautbarungen des WDR zum Trotz. Sie befanden sich damit freilich in voller Übereinstimmung mit ihrem Hauptabteilungsleiter Hans-Geert Falkenberg, der bei den Mainzer Tagen der Fernsehkritik 1971 für das Glashaus einen »kompromißlos harten Kurs in der intramedialen Kritik des Fernsehens " gefordert hatte und der es ausdrücklich als Schwäche bisheriger Versuche bezeichnet, daß sie »eher didaktisch als kritisch « daherkamen. ${ }^{1}$ Falkenberg führte sogar Beispiele vor, so aus dem Programm der BBC, um die dort praktizierte Härte der Kritik zu demonstrieren.

Dr. Rainald Merkert ist Akademischer Oberrat und Privatdozent am Erziehungswissenschaftlichen Institut der Universität Düsseldorf 
Die von ihm geforderte und von der Glashaus-Redaktion dann auch weitgehend praktizierte Kritik des Fernsehens durch das Fernsehen«, häufig auch als Selbstkritik des Fernsehens bezeichnet und mit Transparenz gleichgesetzt, erwies sich jedoch schnell als undurchführbar. Eine Institution kann sich nämlich gar nicht selbst kritisieren. In Wahrheit sind es immer nur einige wenige, die die vielen anderen kritisieren, die Verantwortlichen des Hauses nicht ausgenommen. So ist es nur eine Frage der Zeit, bis die hauseigenen Kritiker aus der Sicht der Kritisierten in der Rolle von Grabenkämpfern und Partisanen, von Widersachern in den eigenen Reihen erscheinen und auch als solche behandelt werden. Insofern sind aller Fernsehselbstkritik sehr enge Grenzen gesetzt. Das ändert sich auch dadurch nicht, daß man Zuschauer als Kritiker zu Wort kommen läßt, denn diese müssen ja vom verantwortlichen Redakteur ausgewählt und "zugelassen " werden.

größere Unabhängigkeit der Programmarbeit garantiert ist, aber offensichtlich sind die Widerstände dennoch zu stark. Auch Falkenberg hat diese Widerstände institutioneller wie psychologischer Art gesehen, war aber optimistisch genug, sie für überwindbar zu halten. Zu nennen sind etwa der Ressortegoismus innerhalb der Anstalten, der dazu führt, daß jede Redaktion, Abteilung und Hauptabteilung »eine elementare Kritik ihres Programms im Programm«als Schwächung ihrer Position ansieht. Zu nennen ist die naive Anpassung an den Erfolg, wie er sich in Zuschauerzahlen niederschlägt, und die kritiklose Identifikation mit dem eigenen Programm - eine Bewußtseinslage, die der der kommerziellen Systeme sehr ähnlich ist. Diese Identifikation wird zudem gefördert durch die Anonymität und Isolation der Programmarbeit, was manchen Redakteur dazu verführt, Sendungen mehr für die Kollegen oder gar für sich selbst zu machen als für das Massenpublikum. Und schließlich ist auf das merkwürdige Phänomen hinzuweisen, daß Journalisten, deren Geschäft neben dem Bericht die Kritik ist, überaus empfindlich sind gegen jegliche Kritik, die sie selber trifft - aus welchen Gründen auch immer.

Das ZDF war von vornherein behutsamer. Dort bezeichnete man die Sendereihe »betrifft: fernsehen« als medienkundliche Reihe. Sie existierte vom März 1974 bis März 1984 und siebrachte nach Auskunft des ZDF-Jahrbuchs in diesem Zeitraum exakt fünfzig »medienkundliche Sendungen «. Ihr Redakteur war Helmut Greulich, zugleich einer der ganz wenigen Programmacher, die sich schon in den sechziger Jahren im Fernsehen mit dem Fernsehen befaßt hatten. Er versuchte, weniger die Kritik zu forcieren, vielmehr tatsächliche Medienkunde zu betreiben. Wenn man jedoch davon ausgeht, daß journalistische Praxis angesiedelt ist zwischen Bericht und Kritik, den »beiden zentralen Spannungspolen der Journalistik «, ${ }^{2}$ dann ergibt sich schon daraus, daß auch bei dem Journalisten Greulich das kritische Moment nicht fehlte. Der Beschränkung auf die bloße "Kunde« ist zudem eine ebenfalls unerwünschte, weil der Transparenz entgegenstehende affirmative Tendenz eigen: es wird informiert über das, was ist, über das bestehende System und seine Arbeitsweise also, und das läuft vor dem Hintergrund der skizzierten Produzentenmentalität dann in der Regel auf die Glorifizierung der eigenen Leistungsfähigkeit hinaus. Beispiele dafür gibt es genug. Von dieser Art wollten die medienkundlichen Sendungen des ZDF nicht sein.

Doch trotz des Verzichtes auf provozierende Kritik war Greulichs Medienkunde offenbar immer noch zu kritisch. Jedenfalls war schon im März 1979 beim zweiten Stuttgarter Fachgespräch über Medienpädagogik zu hören, daß die bisherige Arbeit der Redaktion an einem toten Punkt angekommen sei. Programmanalytische ebenso wie produktionsbeobachtende Sendungen seien praktisch nicht mehr möglich, weil die Kollegen für eine Analyse kein Material mehr herausgäben und auch mit einer Produktionsbeobachtung nicht mehr einverstanden seien. ${ }^{3}$ Nicht die Anstaltshierarchen also, sondern die Kollegen blockten ab; ihrer Mentalität erschienen offenbar kritische Urteile sogleich als Verurtei- 
lungen. Etwa zur gleichen Zeit prangte an Greulichs Bürotür im ZDF eine Zeitungsanzeige, in der ein »Kanalreinigungsunternehmen Greulich $*$ Geschäftsreklame machte. Zweifellos wollte sich da ein Kollege nur einen Scherz erlauben, aber es war ein aufschlußreicher Scherz.

Auffällig ist, daß bei WDR wie ZDF sorgfältig die Bezeichnung »Medienpädagogik « vermieden wurde. Der Grund dafür ist eindeutig: Journalisten wollen in der Regel nichts weniger sein als Pädagogen; will man ihrer Vermittlungstätigkeit mit pädagogischen beziehungsweise didaktischen Kategorien zuleibe rücken, so reagieren sie allergisch; für Produzenten wie Kritiker bedeutet die Qualifizierung einer Sendung als Schulfernsehen nach wie vor ein negatives Urteil, so töricht das ist. (Daß viele gleichwohl sich höchst "pädagogisch $*$ gebärden, mitunter geradezu als Lehrmeister der Nation, steht auf einem anderen Blatt und läuft bei ihnen unter der Bezeichnung »Engagement “.) So ist es nur konsequent, daß nach dem Sprachgebrauch der Anstalten medienpädagogische Sendungen nur in Schulfernsehen und Schulfunk angesiedelt sind. Tatsächlich werden von diesen Redaktionen mit einer gewissen Regelmäßigkeit, selbstverständlich mit Unterschieden zu Anstalt zu Anstalt, qualifizierte Sendungen angeboten. ${ }^{4}$ Eine Sonderstellung nimmt der SDR ein, der eine eigene Hörfunk-Redaktion Medienpädagogik eingerichtet hat.

Doch soll hier nicht ein umfassender Rück- und Rundblick gegeben werden, sonst müßte man auch auf die einstigen Bemühungen des SWF-Fernsehens eingehen, das zunächst in den siebziger Jahren unter dem Reihentitel »Mikado «, später als ein-oder mehrteilige Einzelsendungen einschlägige Programme ausstrahlte. Dabei lag der Akzent vorwiegend auf der pädagogischen Problematik, die das Fernsehen für die Familie, insbesondere für die Kinder mit sich bringt. Aber auch verschiedene ZDF-Sendungen müßten dann erwähnt werden, zum Beispiel aus der Reihe "Kinder Kinder«.

Festzuhalten ist hingegen noch ein anderes Dilemma, für das unter anderem ein Kurzartikel in der Funk-Korrespondenz vom 17. August 1983 symptomatisch ist. Unter der Überschrift »Kein Mut zur Selbstkritik« wird übèr das bevorstehende Ende von »betrifft: fernsehen * berichtet. Auf der ersten Zeile wird diese Reihe als medienkritische, auf der vierten als medienpädagogische bezeichnet; im weiteren ist dann von selbstkritischen Sendungen die Rede, von einem medienkundlichen Ansatz, von Medientransparenz und kritischer Selbstdarstellung. Nur einmal wird ansatzweise zwischen Kritik und Kunde unterschieden, sonst fehlt jegliche terminologische Differenzierung, die Autorin tut so, als handle es sich bei den benutzten Begriffen im Grunde um Synonyme. Das ist durchaus charakteristisch für die Diskussion: man stößt kaum einmal auf die ntowendige begriffliche Präzision, derselbe Begriff wird von verschiedenen Autoren unterschiedlich gebraucht, andererseits kann mit verschiedenen Begriffen durchaus dasselbe gemeint sein. So aber redet man nicht nur aneinander vorbei, es werden auch höchst disparate Erwartungen geweckt, die keine Redaktion erfüllen kann.

\section{Transparenz und Bildung}

Doch soll auch auf dieses terminologische Problem hier nur am Rande hingewiesen, es soll aber nicht thematisiert werden. Im folgenden soll vielmehr auf eine bisher vernachlässigte, gleichwohl wesentliche Perspektive der Forderung nach Transparenz aufmerksam gemacht werden. Die These soll vertreten werden, daß Medientransparenz vor allem eine Bildungsaufgabe ist, daß sie folglich als eine solche auch anzugehen ist, außerhalb wie innerhalb der Medien. Das Transparentmachen des medialen Charakters der Medien, dessen Aufzeigen oder Aufklären mag dann stärker medienkundlich oder medienkritisch 
oder medienpädagogisch akzentuiert sein, entscheidend ist, daß es auf die Ermöglichung von Bildung hin orientiert ist.

Was aber hat Medientransparenz mit Bildung zu tun? Und ist nicht der Bildungsbegriff so diffus und vieldeutig, überdies historisch belastet, daß er hier am wenigsten weiterhelfen kann? Glücklicherweise ist es jedoch zur Erläuterung unserer These nicht notwendig, auf bildungstheoretische Kontroversen einzugehen. Denn bei aller Verschiedenheit theoretischer Positionen besteht durchaus Einmütigkeit darüber, daß Bildung wesentlich Weltverständnis und Weltorientierung des Menschen umschließt. "Eine jede Bildungstheorie muß aber von dem Verhältnis des Menschen zur Welt ausgehen, jener Urrelation, die für Bildung überhaupt konstitutiv ist. « ${ }^{5}$

Grundlegend für unsere Zeit ist nun die Tatsache, daß diese Urrelation von Mensch und Welt durch die Entwicklung der modernen Medien und deren tägliche Nutzung entscheidend verändert wird. Die Wirklichkeit der Welt, von Natur, Kultur, Gesellschaft, selbst Kirche, wird immer weniger direkt und unmittelbar, immer weniger im eigentätigen Umgang erfahren, sie wird zunehmend durch Medien vermittelt. Dies ist zunächst nur als Feststellung einer Tatsache gemeint und nicht, wie heute fast die Regel, bereits als ein Urteil. Um dieser Feststellung Anschaulichkeit zu geben, könnte man die heutigen Gegebenheiten etwa mit denen des Mittelalters in Beziehung setzen, wie Otto Borst sie darstellt in seinem Buch Alltagsleben im Mittelalter «, das ein eigenes Kapitel »Kommunikation ohne Vervielfältigung « enthält. ${ }^{6}$

Soll geurteilt werden, so muß man gründlich abwägen. Der Mensch als körperlichsinnlich-geistiges Lebewesen ist zunächst ein Wesen der Nähe, das heißt er macht die für seine gesamte Weltorientier ung grundlegenden Erfahrungen im Umgang mit Menschen und Dingen seiner unmittelbaren Umgebung, er macht sie, "so weit das Auge reicht". Durch die Vermittlung moderner Medien wird sein Horizont enorm erweitert, freilich um den Preis, daß diese erweiterte Welterfahrung nur eine indirekte, eine medienvermittelte ist. Bekanntlich wird darüber gestritten, ob dies nicht eher eine Pseudoerfahrung sei, die deshalb eher der Desorientierung als dem Weltverständnis diene. In bildungstheoretischer Perspektive ist hier zweierlei von Belang.

1. Während die Erziehung des Erziehers bedarf, ist Bildung wesentlich Selbstbildung, das heißt Ergebnis der Eigentätigkeit, die durch niemanden abgenommen werden kann. Wenn Medieninhalte nur als Neugierfutter rezipiert werden, können die nicht das Weltverständnis und damit auch das Selbstverständnis erweitern oder gar vertiefen. Das ist nur möglich, wenn, wie Borst es formuliert, "aktive Nachrichtenaufnahme " praktiziert wird anstatt bloß »passiver Neuigkeiten-Hinnahme«. Die produktive Reaktion, das Verarbeiten der Medieninhalte ist unerläßlich, soll durch Mediennutzung ein Zuwachs an Welterfahrung stattfinden.

Dieses Verarbeiten kann nur erfolgen auf der Basis bereits vorhandener Erfahrung, es ist also von ihr abhängig. Zum anderen ist es abhängig von der Aufnahme- und Verarbeitungskapazität des Mediennutzers, der intellektuellen wie emotionalen. Für eine realistische Anthropologie ist es deshalb keineswegs verwunderlich, daß von den meisten $\mathrm{Zu}$ schauern nur wenige Inhalte tatsächlich aufgenommen werden, relativ umso weniger, je mehr sie fernsehen. - Von diesen Tatbeständen aus müßte übrigens auch der Begriff der Aktualität neu gefaßt werden. Seine Herkunft vom lateinischen agere weist bereits darauf hin, daß das Aktuelle in Beziehung steht zur aktiven Informationsaufnahme, mehr noch, daß nicht die neueste Neuigkeit aktuell ist, sondern was in Beziehung steht zum Handeln der Mediennutzer. Mit vielen Programmen weiß der Zuschauer nichts anzufangen, weil 
sie viel zu weit weg sind von seiner Welt und seinem Leben. Dieses dürfte eine entscheidende Schwäche unseres gesamten Medienwesens sein, daß die Mehrheit seiner Inhalte einschließlich der Tagesnachrichten zu wenig zu tun hat mit den Handlungs- und Entscheidungsmöglichkeiten seiner Nutzer; deshalb kommen sie gar nicht bei ihnen an oder sind im Handumdrehen wieder vergessen.

2. Für unser Thema ist ein anderer Aspekt wichtiger. Wenn die massenmedial vermittelten Informationen der Weltorientierung dienen sollen, dann muß man etwas von der Eigenart der Vermittlungsprozesse verstehen. Früher sagte man mit Blick auf die Zeitung, wer von ihrer Lektüre profitieren wolle, der müsse zwischen den Zeilen lesen können. Das auf dem Bildschirm gebotene kann die Wirklichkeit erhellen, es kann sie aber auch verstellen; es ist nicht die Wirklichkeit selber, sondern immer eine schon selektierte, interpretierte, manipulierte Wirklichkeit, manipuliert im zunächst wertfreien Sinn dieses Wortes. Mehr noch, Wirklichkeit wird durch Medien nicht nur wiedergegeben, sie wird durch sie zugleich definiert und konstituiert; ${ }^{7}$ was die Tagesschau an Tagesaktualitäten bringt, wird eben dadurch für die allermeisten Zuschauer überhaupt erst zur Tagesaktualität. Oder noch einmal anders gesagt: Wenn die These von der gesellschaftlichen Konstruktion der Wirklichkeit, wie Berger und Luckmann sie vertreten, ${ }^{8}$ zutrifft, dann liegt auf der Hand, daß die Medien der gesellschaftlichen Kommunikation an der Konstruktion von Wirklichkeit kräftig mitwirken.

Mit diesen knappen Sätzen ist eine Fülle konkreter Gegebenheiten angesprochen, die den Vermittlungsprozeß beeinflussen: technische und dramaturgische, institutionelle und organisatorische, juristische und politische, ökonomische und psychologische, berufskulturelle und ideologische. Wer von diesen Einflüssen und ihrer jeweils konkreten Gestalt keinerlei Ahnung hat, der kann sich zwar trotzdem gut unterhalten, aber er kann sich nicht gut informieren. Medientransparenz nun bedeutet, ein Medium in seinen vielfältigen Bedingtheiten so durchsichtig zu machen, daß der Blick tatsächlich in etwa frei wird auf die durch Bild und Wort vermittelte, zugleich durch das Medium gebrochene Wirklichkeit . Wie kann zum Beispiel eine Sendung der Weltorientierung dienen, Welt verstanden im Sinne der großen internationalen Welt, wenn der Zuschauer nicht weiß und auch nie erfährt, unter welchen Bedingungen und Auflagen des betreffenden Landes sie entstanden ist. Die große Mehrheit der Zuschauer neigt ja im Gegenteil dazu, etwa nach dem Muster der Zusammenschnitte der Fußball-Bundesliga, dem Objektiv der Kamera mehr zu vertrauen als den eigenen Augen oder denen des Schiedsrichters.

Medien transparent zu machen, ist sicher auch ein kritisches Geschäft, aber Kritik ist dabei nicht die primäre Zielsetzung. Nicht um das Anprangern der Grenzen des Mediums und seiner Mitarbeiter geht es, auch nicht um deren Uberheblichkeit oder Dilettantismus, so sehr das mitunter angebracht sein mag, sondern um Medientransparenz zum Zwecke der Förderung von Weltverständnis und Weltorientierung. Eben dieses ist mit der These gemeint, daß Medientransparenz eine genuine Bildungsaufgabe darstellt.

Sie stellt sich folglich allen Bildungsinstitutionen, von der Schule bis zur Erwachsenenbildung. Sie stellt sich aber auch dem öffentlich-rechtlichen Fernsehsystem, insofern ihm nämlich neben Unterhaltung und Information auf Bildung aufgetragen ist. Wenn oben festgestellt wurde, daß diese öffentlich-rechtliche Verfassung Medientransparenz möglich mache, so ist jetzt hinzuzufügen, daß sie sie zugleich erforderlich macht. Es gehört zu den Merkwürdigkeiten dieses Systems, daß es sich mit allem und jedem befaßt, nur nicht mit sich selbst; daß man Rat gibt für fast alle Lebenslagen, nur nicht für jene, die das eigene Medium heraufbeschworen hat; daß bisherige mehr oder minder mißglückte Ansätze kein Ansporn waren zu neuen Versuchen, sondern eher Anlaß zur Resignation; daß 
in diese zwar nicht leicht zu lösende, aber doch wichtige und auch interessante Bildungsaufgabe bisher erstaunlich wenig Fantasie investiert worden ist. Einer der Gründehierfür mag sein, daß man den Bildungscharakter dieser Aufgabe bisher nicht deutlich genug gesehen hat. Tatsächlich zeigt sich in Gesprächen, daß für viele Fernsehleute Medientransparenz im Grunde auf Warnung vor dem eigenen Medium und seinen Gefahren hinausläuft.

Diehier vertretene These mag auf den ersten Blick völlig unzeitgemäß erscheinen. Die gegenwärtigen Entwicklungen im Medienbereich, auch innerhalb der öffentlichrechtlichen Anstalten, scheinen höchst ungeeignet, gerade diese Bildungsaufgabe wahrzunehmen, wird doch schon der Bildungsauftrag generell immer stiefmütterlicher behandelt. Von mehr Programm ist die Rede, aber nicht von besserem Programm, erst recht nicht davon, wie man dem Zuschauer zur besseren Nutzung von Programm im Sinne erweiterter und vertiefter Weltorientierung verhelfen kann. Andererseits jedoch zeigen diese gegenwärtigen Entwicklungen, welche wichtige Bildungsaufgabe Medientransparenz ist und welche Folgen bisherige Versäumnisse haben. Man muß ja nicht gleich die Massen erreichen wollen; zwar braucht Bildung keineswegs elitär zu sein, wohl aber erfordert sie wie stets so auch in diesem Falle ein Stück eigener geistiger Arbeit. Dieses in Erinnerung zu rufen, ist angesichts der gegenwärtigen Medienpolitik in der Tat unzeitgemäß, deshalb aber umso notwendiger.

\section{Anmerkungen}

1 Hans-Geert Falkenberg: Die Kritik des Fernsehens durch das Fernsehen. In: Bernward Frank (Hrsg.): Fernseh-Kritik, Mainz 1972, S. 118.

2 Josef Derbolav: Pädagogik und Politik. Stuttgart 1975, S. 149.

3 Vgl. Funk-Korrespondenz 11/1979, S. 17 f.

4 Vgl. etwa Lothar Humburg: Medienpädagogik in der Praxis. In: medienpraxis, Grundlagen 2, hrsg. von der Zentralstelle Medien, Bonn 1985, S. 50 f.

5 Clemens Menze: Bildung. In: Josef Speck/Gerhard Wehlte (Hrsg.): Handbuch pädagogischer Grundbegriffe I, München 1970, S. 159.

6 insel Taschenbuch 513, Frankfurt 1985, S. 530-562.

7 Vgl. Rainald Merkert: Fernsehen und Wirklichkeit. In: „Stimmen der Zeit«200: 1982, S. 483-495.

8 Peter L. Berger/Thomar Luckmann: Die gesellschaftliche Konstruktion der Wirklichkeit, Fischer Taschenbuch 6623, Frankfurt 1980. 


\section{SUMMARY}

The TV series "Glashaus« (Glass House) failed because it did not prove transparent as its title had promised but showed criticism of the television instead.

Criticism of the television by the institution itself, however, is strictly limited as those who are criticised oppose to it. On the other hand the second part of the essay deals with the thesis that the transparency of the media is an educational task in the first place. Education is concerned with man's relation to the world. However, this basic relation between man and world which is constitutive for education has been decisively changed by the modern media and their daily consumption: The world is communicated to an increasing extent by means of the media. It is true that those for whom this process of communication is not transparent in its conditionality are nevertheless able to converse well with others, but their comprehension of and orientation in the world is scarcely extended by the media. But, if the transparency of the media is a genuine educational task, also television as a public institution which has to communicate education besides information and entertainment has to accept the challenge.

\section{RÉSUMÉ}

La série d'émissions «Glashaus« a été un échec parce qu'au lieu de respecter la transparence proposée par son titre, elle s'est livrée à une critique de la télévision. La critique de la télévision par la télévision est très limitée parce que ceux qui sont ainsi critiqués s'y opposent. Par contre, dans la deuxieme partie de l'exposé, on soutient la thèse que la transparence des média est un devoir primaire du point de vue éducatif. L'éducation a pour tâche d'influencer le comportement de l'homme vis à vis de la société. Cette relation fondamentale de l'homme avec la société est un élément constitutif de l'éducation et ne peut être influencée de facon décisive que par des moyens de communication moderne et leur utilisation régulière. La conception du monde est de plus en plus transmise par les média. Ceux dont les limitations ne rendent pas ces rpocédés de transmission transparents peuvent cependant être distraits agréablement, les média ne pourront qu'avec peine augmenter leur compréhension et élargir leurs horizons. Si la clarté des média est vraiment une tâche éducative, c'est aussi le devoir de la télévision de droit public, en plus de l'information et du divertissement, de se charger de l'éducation.

\section{RESUMEN}

La serie televisiva "Casa de Vidrio « fracasó, porque no ofreció la transparencia prometida a través de su nombre, sino que en lugar de ello, crí tica a la televisión misma. La crí tica de la televisión por la televisión tiene sus l ímites, porque los criticados se oponen a ella. En la segunda parte del art ículo, se sostiene la tesis contraria de que la transparencia de los medios de comunicación, es primariamente una tarea educativa. La educación tiene que ver con la relación del hombre con el mundo; esta relación elemnental del hombre y el mundo, que es constituyente de la educación, será modificada decididamente por los medios de comunicación modernos y su uso diario: el mundo es presentado progresivamente a través de los medios de comunicación. Quién no entrevea este proceso de mediación en su relatividad, puede entretenerse bien, pero su comprensión y orientación del mundo, se ampliará muy dificultosamente con los medios de comuncación. Si la transparencia de los medios de comunicación es una tarea genuina de la educación, entonces la televisión pública corporativa tiene que encargarse de la educación, además de la información y la entretención. 